\title{
Numerical investigation of dynamic effects for sliding drops on wetting defects
}

\author{
Andrea Cavalli, ${ }^{1}$ Michiel Musterd, ${ }^{2}$ and Frieder Mugele ${ }^{1, *}$ \\ ${ }^{1}$ Physics of Complex Fluids and MESA+ Institute for Nanotechnology, University of Twente, 7522 NB Enschede, The Netherlands \\ ${ }^{2}$ Product and Process Engineering, TNW-PPE/TP, Delft University of Technology, 2628 CN Delft, The Netherlands
}

(Received 6 August 2014; published 17 February 2015)

\begin{abstract}
The ability to trap or deflect sliding drops is of great interest in microfluidics, as it has several technological applications, ranging from self-cleaning and fog harvesting surfaces to laboratory-on-a-chip devices. We present a three-dimensional numerical model that describes sliding droplets interacting with wetting defects of variable strength and size. This approach provides relevant insight if compared to simplified analytic models, as it allows us to assess the relevance of the internal degrees of freedom of the droplet. We observe that the deformation of the drop enhances the effective strength and range of the defect, and we quantify this effect by comparison to a point-mass model. We also analyze the role of the steepness and strength of the defect on the drop motion, observing that small, strong defects are more effective at trapping than large, shallow traps of same excess surface energy. Finally, our results show quantitative agreement with previously reported electrowetting experiments, suggesting a universal behavior in droplet trapping that does not depend strongly on the nature of the defect.
\end{abstract}

DOI: 10.1103/PhysRevE.91.023013

PACS number(s): 47.90.+a, 68.08.-p

\section{INTRODUCTION}

The interaction of sessile drops with microscopic chemical or topological defects is of great interest, as the well-known phenomenon of contact angle hysteresis is due to the pinning of the contact line on such obstacles [1,2]. Different authors have analyzed the onset of motion for sessile droplets under the action of gravity [3-6], as well as the depinning transition from defects in lubrication approximation [7]. The motion of sessile drops on periodically patterned substrates [8-12] has attracted a particular interest, since the ability to shed droplets is one of the main design objectives for micropatterned, liquid repellent surfaces [13]. Moreover, wetting defects can find several applications in microfluidics devices as a means to steer or retain droplets in microchannels [14-16]. However, such defects usually occur in a wide range of shapes, sizes, and strengths, making a systematic analysis of the interaction with a single defect complicated [17,18]. A significant step forward in these directions is the "traps" designed by 't Mannetje et al. [16], which exploit electrowetting to realize defects of tunable strength. In order to extend our understanding of the interaction of a sliding drop with a wetting defect, we introduce here a three-dimensional numerical approach. Our goal is to analyze in detail the kinematics and dynamics of the interaction in order to assess the importance of different parameters, such as surface tension, inertia, and the wettability contrast of the substrate. Our model allows us to properly account for internal degrees of freedom and deformations in the drop, which play a significant role in determining the escaping or trapping threshold for a given wetting defect. This gives us relevant information which is not captured by simplified analytic approaches. Another feature of our model is the ability to address defects of different kind in a unified framework, thus observing how different parameters (strength, size, and steepness of the obstacle) affect the trapping. Finally, after a simple normalization step, we are able to compare

\footnotetext{
${ }^{*}$ To whom correspondence should be addressed: f.mugele@utwente.nl
}

our simulations to previously reported electrowetting trapping experiment, observing a quantitative agreement.

\section{NUMERICAL METHODS}

We implement our model using the open-source computational fluid dynamics software OPENFOAM. We choose the volume-of-fluid solver "interFoam," which implements the incompressible Navier-Stokes equations for two immiscible fluids, using a diffuse interface approach. Detailed information about the implementation and performance of the solver can be found in Ref. [19]. Briefly, we define a field $\psi(\mathbf{x}, t)$, which describes the fraction of each computational cell occupied by the liquid phase:

$$
\psi(\mathbf{x}, t)=\frac{1}{\Omega_{i}} \int_{\Omega_{i}} \chi_{\mathrm{drop}}(\mathbf{x}, t) d V .
$$

Here $\Omega_{i}$ is the volume of the $i$-th computational cell and $\chi_{\text {drop }}(\mathbf{x}, t)$ is the indicator function of the liquid phase, which is equal to 1 inside the drop and 0 outside. The volume fraction field is advected by the flow velocity $\mathbf{u}$, resulting in the equation of motion

$$
\frac{d \psi}{d t}+\nabla \cdot(\mathbf{u} \psi)=-\nabla \cdot\left(\mathbf{u}_{\mathbf{c}} \psi(1-\psi)\right) .
$$

Equation (2) preserves the liquid and gas volumes inside the simulation domain and generates smooth $\psi$ profiles, where the transition from liquid $(\psi=1)$ to gas $(\psi=0)$ phase is smeared over a finite thickness that can be tuned by the parameter $\mathbf{u}_{\mathbf{c}}$ [19]. The dynamics described by Eqs. (1) and (2) has been extensively applied to two-phase flow problems $[19,20]$. It is worth mentioning that the diffuse interface is a numerical approximation, meant to avoid discontinuous jumps in the physical parameters. The dynamics of the system is, however, not significantly affected, as long as the thickness of the interface is small in comparison to the other length scales in the simulation, i.e., comparable to the resolution of the computation mesh. Fluid properties relevant to the flow, such as density and viscosity, are interpolated across the 
interface as

$$
\begin{aligned}
& \rho=\psi \rho_{\mathrm{liq}}+(1-\psi) \rho_{\mathrm{gas}}, \\
& \mu=\psi \mu_{\mathrm{liq}}+(1-\psi) \mu_{\mathrm{gas}},
\end{aligned}
$$

where $\rho_{\text {liq }}$ and $\rho_{\text {gas }}$ and $\mu_{\text {liq }}$ and $\mu_{\text {gas }}$ are the densities and dynamic viscosities of the liquid and gas phases, respectively. We can see that the bulk properties of the liquid and gas phases are recovered by substituting $\psi=1$ and $\psi=0$, respectively, inside Eqs. (3) and (4). Once more, intermediate values of density and viscosity at the liquid-gas interface are not physical but required for numerical stability and will not affect the outcome as long as the interface thickness is small. Aside from Eq. (2), we need to solve the equations of motion dictated by mass and momentum conservation for the whole system. These are the continuity and Navier-Stokes equations,

$$
\nabla \cdot \mathbf{u}=0
$$

$$
\begin{aligned}
\frac{d \rho \mathbf{u}}{d t}+\mathbf{u} \cdot \nabla \rho \mathbf{u}= & -\nabla p+\nabla \cdot\left(\mu\left[\nabla \mathbf{u}+\nabla \mathbf{u}^{T}\right]\right)+\rho \mathbf{g} \\
& +\int_{\text {drop }} \gamma \kappa \mathbf{n} \cdot d \Sigma .
\end{aligned}
$$

Here $\rho$ and $\mu$ are defined according to Eqs. (3) and (4); $\mathbf{u}$ is the velocity field; $p$ is the pressure field; $\mathbf{g}$ is the gravity acceleration; $\gamma$ is the water-air surface tension; $\kappa$ is the local mean curvature of the interface, which can be expressed in terms of the volume fraction field as $\kappa=\nabla \cdot \mathbf{n}=\nabla \cdot \frac{\nabla \psi}{|\nabla \psi|}$; and $\mathbf{n}$ is the unit normal to the interface. The last term on the right-hand side can then be recognized as the Young-Laplace contribution to the fluid pressure.

\section{GEOMETRY AND SETUP}

In Fig. 1 we sketch the setup of our system. Unless otherwise specified, we consider sessile water drops in air of volume $\mathrm{V}=40 \mu \mathrm{L}$. Our simulation domain is a rectangular box of $1.5 \times 0.5 \times 0.5 \mathrm{~cm}$. A stripe of width $\mathrm{a}=0.5 \mathrm{~mm}$ (in yellow in the sketch), whose wettability can be tuned, runs along the $z$ direction, across the domain. We exploit the symmetry of the system with respect to the midplane of the drop $(z=0)$ to simulate only half of it. We apply a periodic boundary condition in the $x$ direction (the striped boundaries in the figure are mapped on each other). Finally, we impose a no-slip boundary condition on all other (nonsymmetric, nonperiodic) boundaries. The drop slides under the action of the gravity force $\mathbf{F}_{\mathbf{g}}=(\mathrm{mg} \sin \alpha, \mathrm{mg} \cos \alpha, 0)$, where $\alpha$ is the

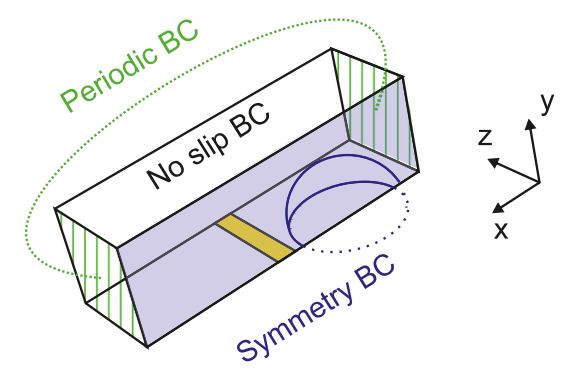

FIG. 1. (Color online) Sketch of the computational domain. slope of the substrate. We start each computation by initializing a hemispherical drop in the center of the domain and setting the same contact angle $\theta_{0}=90^{\circ}$ across the whole substrate. We let the drop slide until it reaches terminal velocity (typically 1-2 $\mathrm{s}$ in simulation time or about six passages of the simulation box). We then prescribe a different contact angle $\theta_{\text {trap }}<90^{\circ}$ inside the stripe and observe the interaction of the drop with the defect.

\section{DYNAMIC CONTACT ANGLE MODELING}

The choice of suitable boundary conditions for the contact angle of the sliding drop plays a critical role in our simulations, as the interaction with the substrate eventually determines whether the drop will be trapped by wetting defects.

We developed an OPENFOAM implementation of the CoxVoinov boundary condition [21], which relates the dynamic contact angle $\theta_{\mathrm{CV}}$ for a moving drop to the velocity of the contact line $v_{\mathrm{cl}}$ through the capillary number $\mathrm{Ca}=\frac{\mu_{\mathrm{liq}} v_{\mathrm{cl}}}{\gamma}$ as

$$
\theta_{\mathrm{CV}}^{3}=\theta_{Y}^{3}+9 \mathrm{Ca} \ln \left(\frac{L_{\max }}{L_{\min }}\right) .
$$

This relation can be derived in lubrication approximation. It is based on a smooth transition from the static contact angle $\theta_{Y}$, on the nanometer scale $L_{\min }$, to the macroscopic value $\theta_{\mathrm{CV}}$ at length scale $L_{\max }$, which is observed experimentally. However, applying Eq. (7) as our boundary condition results in a systematic overestimation of the terminal velocity of the droplets if compared to experimental measurements. This is most likely due to neglected dissipation sources in our model, such as adsorption and desorption processes at the molecular level in proximity of the contact line. One way to account for these effects is to define an alternative dynamic contact angle $\theta_{\text {micro }}$, as suggested, among others, by Petrov [22]:

$$
\theta_{\text {micro }}=\arccos \left[\cos \theta_{Y}-\frac{2 k_{B} T}{\gamma \lambda_{A}^{2}} \operatorname{arcsinh}\left(\frac{v_{\mathrm{cl}}}{2 \nu \lambda_{A}}\right)\right] .
$$

Here $k_{B} T$ is the thermal energy of the system, $\lambda_{A}$ is the distance between adsorption centers on the substrate in proximity of the contact line, $\gamma$ is the liquid-gas surface tension, and $v$ is a characteristic frequency of oscillation for the molecular motion between absorption centers. It is also possible to combine the two approaches: We then obtain the following expression for the dynamic contact angle $\theta_{D}$ [22]:

$$
\theta_{D}^{3}=\theta_{\text {micro }}^{3}+9 \mathrm{Ca} \ln \left(\frac{L_{\max }}{L_{\min }}\right)
$$

In Fig. 2(b) we plot the dynamic contact angle according to the different models discussed for increasing values of the static contact angle $\theta_{Y}$. The dynamic contact angle can be larger or smaller than $\theta_{Y}$, depending on the sign of the contact line velocity: In this way, Eq. (9) describes the dynamics of the contact line in a unified approach. It is also worth noticing that in our model the advancing and receding contact angles (for static droplets) are the same, i.e., there is no prescribed static hysteresis. In Fig. 2(a), we compare the terminal center-of-mass velocity $v_{\text {c.m. }}$ of the droplet, obtained implementing Eq. (9) in the simulation, to experimentally observed values [16] for volumes 
(a)

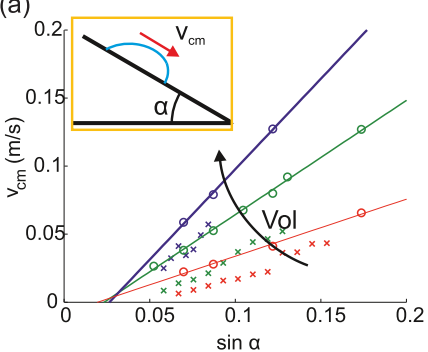

(b)

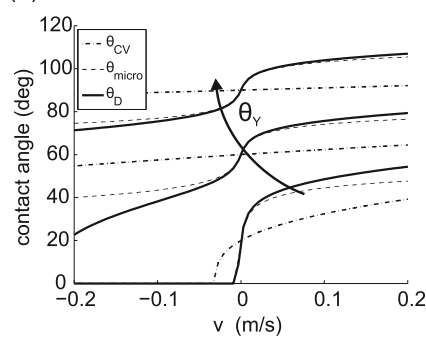

FIG. 2. (Color online) (a) Values of terminal velocities for water droplets of volume $V=20,40,60 \mu \mathrm{L}$ (red, green, and blue sets, with increasing line thickness) as a function of the sine of the slope angle $\sin \alpha$. The circles represents numerical results, while the crosses are experimental measurements from Ref. [16] for water drops on a glass slide covered with an ITO and oil layer, as discussed in the main text. The lines are linear fits to the numerical data. (b) Dynamic contact angles for increasing $\theta_{Y}$, according to the different models described in the main text.

$V=20,40,60 \mu \mathrm{L}$ (red, green, and blue sets, respectively, with increasing line thickness) as a function of the sine of the slope angle $\alpha$. By setting the relevant coefficients as in Table I, we observe a good quantitative agreement. The main difference between our model and the experimental values is a vertical offset in the data. This means that the static hysteresis in the experiments is larger than the one extrapolated from our model. This is expected, as the advancing and receding contact angles are the same in our model, and, consequently, the hysteresis should be zero. The small intercept for the linear fit to the numerical data (corresponding to a slope $\alpha_{0} \simeq 1^{\circ}$ ) is probably due to a nonlinear dependence of the velocity on the slope angle for small $\alpha$. The experiments in Ref. [16] were carried out on a glass substrate covered with an ITO layer and a thin oil film, with advancing and receding contact angles of $\theta_{A}=95^{\circ}$ and $\theta_{R}=92^{\circ}$, respectively. It is worth stressing that, although derived from physical arguments, the coefficients in Eqs. (7) and (8) are effectively used as fitting parameters: A microscopic description of the contact line motion is outside the scope of this paper and should account for the partial cloaking of the water drop by the oil film in the experiments, which would generate extra dissipation at the contact line. The justification of our approach resides ultimately in the agreement with experimental observations.

In terms of numerical implementation, Eq. (9) is used to set the interface normal $\mathbf{n}$ in each computation cell along the contact line of the drop [23]. In order to implement the wetting defects, we locally change the value of $\theta_{Y}$ in Eq. (9) from $\theta_{Y}=\theta_{0}=90^{\circ}$ on the rest of the substrate to $\theta_{Y}=\theta_{\text {trap }}$ inside the defect. As we are interested in discussing the fundamental aspects of the drop-trap interaction, in this paper we address a simple geometry (a stripe defect). It is, however, worth mentioning that our code allows us to specify patches of arbitrary shapes, which will prove useful for microfluidics applications.

\section{RESULTS AND DISCUSSION}

Before discussing the numerical results, we can get a qualitative understanding of the trapping force acting on the drop by considering a simplified picture, i.e., a drop of fixed shape (a hemispherical cap) moving over a patch of prescribed wettability [see Fig. 3(a)]. More specifically, we consider the energy landscape $U(x)$ perceived by a drop sliding over a stripe of width $a$ and prescribed contact angle $\theta_{\text {trap }}, x$ being the distance between the center of the drop and the center of the stripe. In the following, we will denote with $\gamma_{\mathrm{sl}}, \gamma_{\mathrm{sg}}$ the surface tensions between the substrate and the liquid and gas phases, respectively. The corresponding Young contact angle outside the trap is then given by $\cos \theta_{Y}=\cos \theta_{0}=\frac{\gamma_{\mathrm{sg}}-\gamma_{\mathrm{sl}}}{\gamma}$, where $\gamma$ is the liquid-gas surface tension already introduced in Eq. (6). In the same fashion, we will indicate by $\gamma_{\mathrm{tl}}, \gamma_{\mathrm{tg}}$ the surface tensions between the trap and the liquid and gas phases, which results in a contact angle $\cos \theta_{\text {trap }}=\frac{\gamma_{t g}-\gamma_{t l}}{\gamma}$ inside the wetting stripe. In most practical applications, including the modeling in this work, it is sufficient to know $\gamma$ and the contact angles to describe the wettability of the system. Typical values would be $\gamma \simeq 70 \mathrm{mN} / \mathrm{m}$ for water droplets in air at room conditions, while we will consider trap angles in the range $0^{\circ}<\theta_{\text {trap }}<90^{\circ}$. Let $U_{0}$ now be the energy of the droplet when it is completely outside the trap. Once the drop moves over the stripe to a position $x$, its energy becomes:

$$
\begin{aligned}
U(x) & =U_{0}+\left[-\gamma_{\mathrm{sl}}+\gamma_{\mathrm{sg}}\right] A(x)+\left[\gamma_{\mathrm{tl}}-\gamma_{\mathrm{tg}}\right] A(x) \\
& =U_{0}+\gamma\left[\cos \theta_{0}-\cos \theta_{\text {trap }}\right] A(x) .
\end{aligned}
$$

$A(x)$ is the position-dependent overlap between the drop and the stripe,

$$
A(x)=\left\{\begin{array}{ll}
\pi R^{2}-\frac{R^{2}}{2}\left[\phi_{2}-\sin \phi_{2}\right] & -R-\frac{a}{2}<x \leqslant-R+\frac{a}{2} \\
\frac{R^{2}}{2}\left[\phi_{1}-\sin \phi_{1}-\phi_{2}+\sin \phi_{2}\right] & -R+\frac{a}{2}<x \leqslant R-\frac{a}{2} \\
\frac{R^{2}}{2}\left[\phi_{1}-\sin \phi_{1}\right] & R-\frac{a}{2}<x \leqslant R+\frac{a}{2} \\
0 & \text { otherwise }
\end{array},\right.
$$

which can be computed as the difference between two circular segments of opening angles $\phi_{1,2}=\arccos \frac{x \mp a / 2}{R}$ [see Fig. 3(a)].

The change in energy $\Delta U(x)$ and force $F(x)$ acting on the drop are therefore:

$$
\Delta U(x)=\gamma\left[\cos \theta_{0}-\cos \theta_{\text {trap }}\right] A(x),
$$

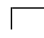

$$
F(x)=-\gamma\left[\cos \theta_{0}-\cos \theta_{\text {trap }}\right] \frac{d A(x)}{d x}
$$

As expected, for our system $\left(\theta_{0}=90^{\circ}\right.$ and $\left.\theta_{\text {trap }}<90^{\circ}\right)$, it is energetically favorable to maximize the overlap between the drop and the stripe. The resulting energy landscape and 
TABLE I. Coefficients employed in the numerical simulation of the dynamic contact angle, as defined in Eqs. (7)-(9).

\begin{tabular}{lcccc}
\hline \hline$L_{\max }$ & Contact angle coefficients & & \\
$L_{\text {min }}$ & $1 \times 10^{-9}(\mathrm{~m})$ & $2.5 \times 10^{-9}(\mathrm{~m})$ & $293.15(\mathrm{~K})$ & $v$ \\
\hline $5 \times 10^{-5}(\mathrm{~m})$ & $1 \times 10^{6}\left(\mathrm{~s}^{-1}\right)$ \\
\hline \hline
\end{tabular}

force are shown in Figs. 3(a) and 3(b), respectively (red thick lines). We can get a simplified expression by noticing that, for thin stripes $a \ll R$, we can approximate $A(x) \simeq 2 a \sqrt{R^{2}-x^{2}}$, from which we get $F(x)=-\gamma \cos \theta_{\text {trap }} \frac{2 a x}{\sqrt{R^{2}-x^{2}}}$ (blue thin lines). The restraining force exerted by the hydrophilic stripe is therefore similar to a non-Hookean spring of constant $k(x)=\frac{2 a \gamma \cos \theta_{\text {trap }}}{\sqrt{R^{2}-x^{2}}}$, and it is in very good agreement with the complete result, with the significant exception of the edges of the drop, where this approximation predicts a divergent rate of change for the droplet-stripe overlap. We also plot a truncated harmonic approximation of the trap profile (green, dashed line) as a reference. Based on Eqs. (11) and (12), we can derive an approximate dynamics for the position $x$ of the center of mass of the drop:

$$
\begin{gathered}
m \ddot{x}+\lambda \dot{x}+F(x)=F_{g}=m g \sin \alpha-m g \sin \alpha_{0} \\
\dot{x}(0)=\frac{F_{g}}{\lambda} \quad x(0)=-(R+a) .
\end{gathered}
$$

Here $m$ is the mass of the drop; $\lambda$ is a dissipation coefficient, accounting for contact line friction and viscous drag; $F(x)$ is the trapping potential defined in Eq. (12); and $F_{g}$ is the gravity force driving the motion, with an offset $\alpha_{0}$ on the sliding angle due to the static hysteretic force acting on the drop. The values of $\lambda=0.0044 \mathrm{~kg} \mathrm{~s}^{-1}$ and $\alpha_{0}=1.33^{\circ}$ are derived from the slope and intercept of the velocity fit reported in Fig. 7 (see also Ref. [16]). This approximation will be useful to distinguish the contribution of internal degrees of freedom to the dynamics of the droplet, and it will allow us to normalize our simulation results and compare them to experiments.

\section{Numerical results}

We will start this section by analyzing the kinematics of the interaction between the sliding droplet and the defect. Figure 4 shows snapshots from a typical simulation using our model for two different combinations of slope $\alpha$ and trap wettability $\theta_{\text {trap }}$. In the top row, we consider a slope of $\alpha=5^{\circ}$ and a defect of $\theta_{\text {trap }}=30^{\circ}$. Under these conditions, the drop is captured by the trap. As expected from the analytic model, the drop accelerates until it is halfway across the stripe and subsequently slows down, as the change in surface energy opposes further movement. By looking at the footprint of the droplet, we can observe a clear discrepancy with respect to the spherical cap approximation, namely the drop stretches along the stripe. This results in an increased effective cross section. Consequently, the interaction of the drop with the defect increases, which makes it harder to escape. In the bottom row, we consider a slope of $\alpha=7^{\circ}$ and a defect of $\theta_{\text {trap }}=45^{\circ}$. Under these conditions the droplet is able to escape thanks to the higher initial velocity and reduced trap strength. However, we still observe a strong pinning of the receding contact line on the stripe, with the drop stretching significantly in the sliding direction before snapping off of the defect.

In order to characterize the trapping or escaping transition, in Fig. 5 we analyze the trapping process as a function of the slope of the surface $\alpha$ and the strength of the defect, which can be parameterized by the difference in contact angle $\Delta \theta_{\text {trap }}=\theta_{0}-\theta_{\text {trap }}$. In all these simulations we keep the width $a$ of the stripe fixed. Green crosses represent droplets escaping the trap, while red circles stand for trapped droplets. A clear transition between the trapping and escaping regimes can be observed, the former appearing for slow drops and strong wetting contrast, while sliding is favored for fast drops or weak defects. The red line represents the escaping or trapping transition predicted by the approximated model of Eq. (13): While qualitatively consistent with the numerical results, it underestimates the strength of the trap and more so for strong wetting defects. To explain this discrepancy, we can look at the energy landscape experienced by the drop in the simulation, which we extract by computing the overlap between the stripe and the drop at different time steps. In Fig. 6(a) we compare (a)

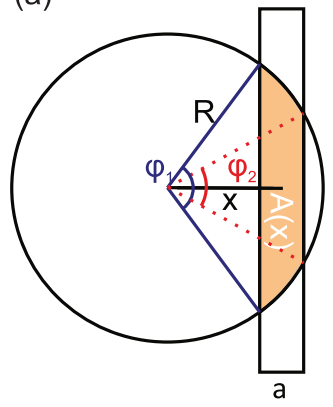

(b)

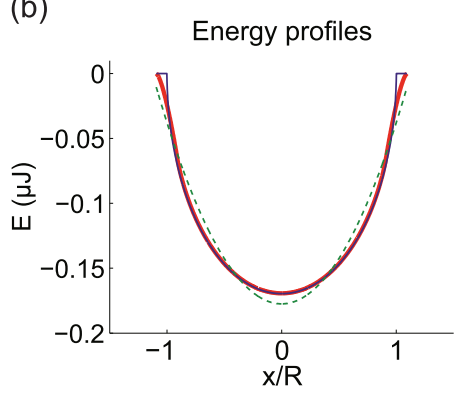

(c)

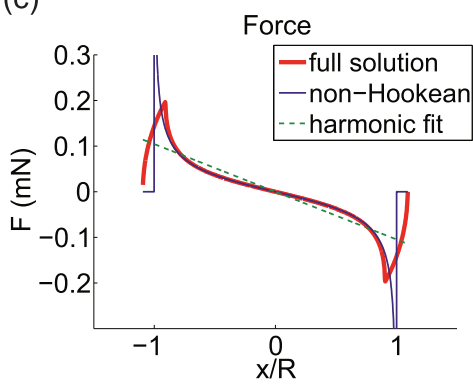

FIG. 3. (Color online) (a) Sketch showing the relevant geometric parameters for the energy and force calculations under the assumption that the drop retains a spherical cap shape. (b) Energy landscape for a 40- $\mu \mathrm{L}$ water drop (spherical cap) sliding over a stripe of width $a=0.5 \mathrm{~mm}$ of contact angle $\theta_{\text {trap }}=45^{\circ}$ as a function of the distance $x$ between the center of the drop and the midpoint of the stripe. (c) Corresponding force acting on the drop as a function of $x$. 

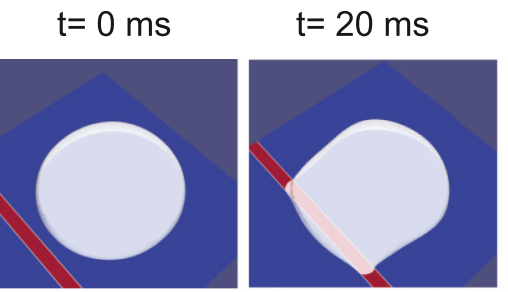

$\mathrm{t}=40 \mathrm{~ms}$

$\mathrm{t}=60 \mathrm{~ms}$

$\mathrm{t}=600 \mathrm{~ms}$
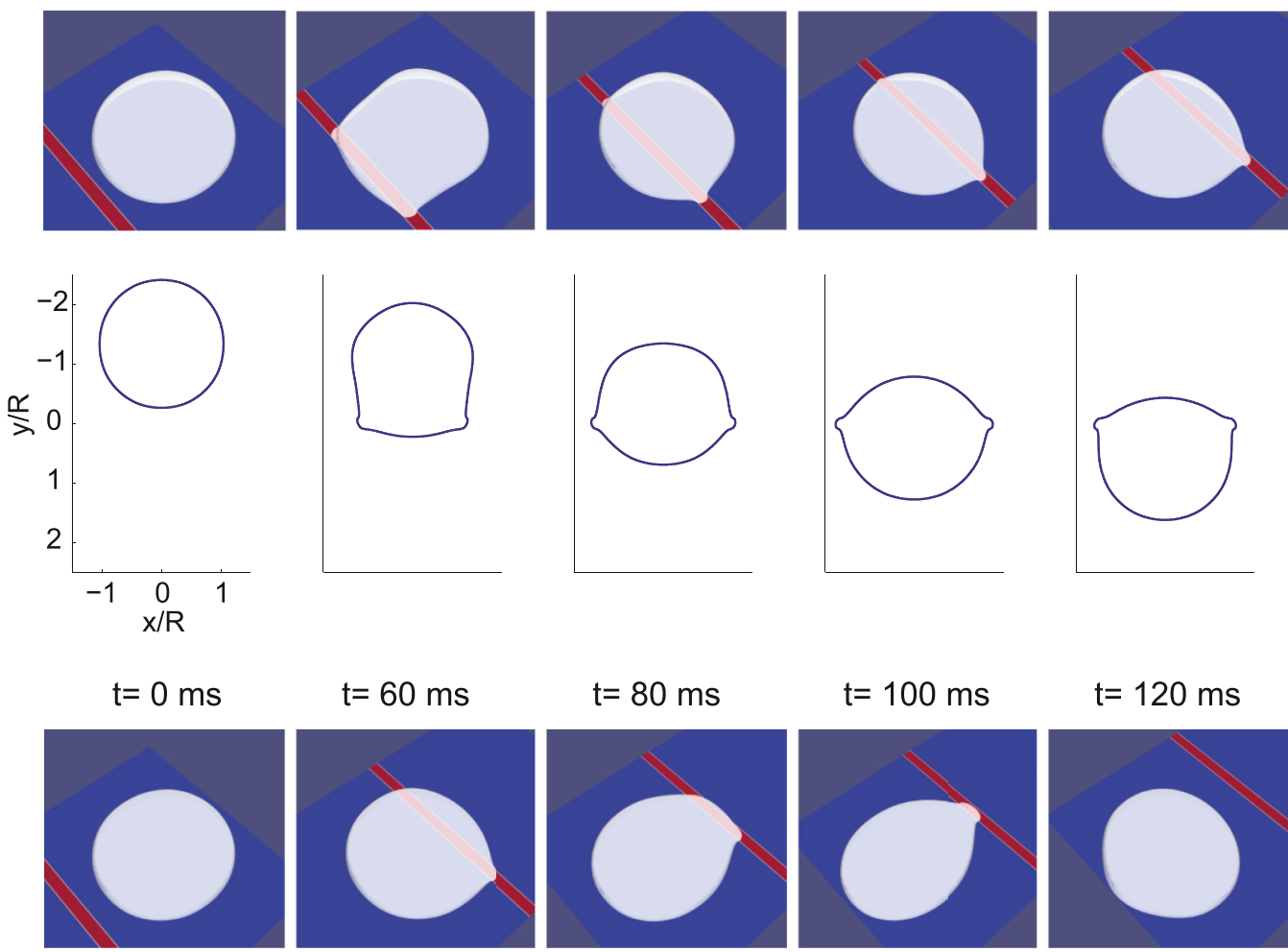

$\mathrm{t}=60 \mathrm{~ms}$
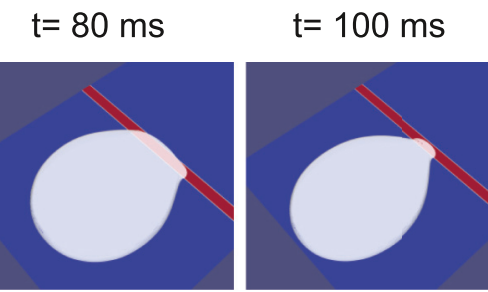

$\mathrm{t}=120 \mathrm{~ms}$
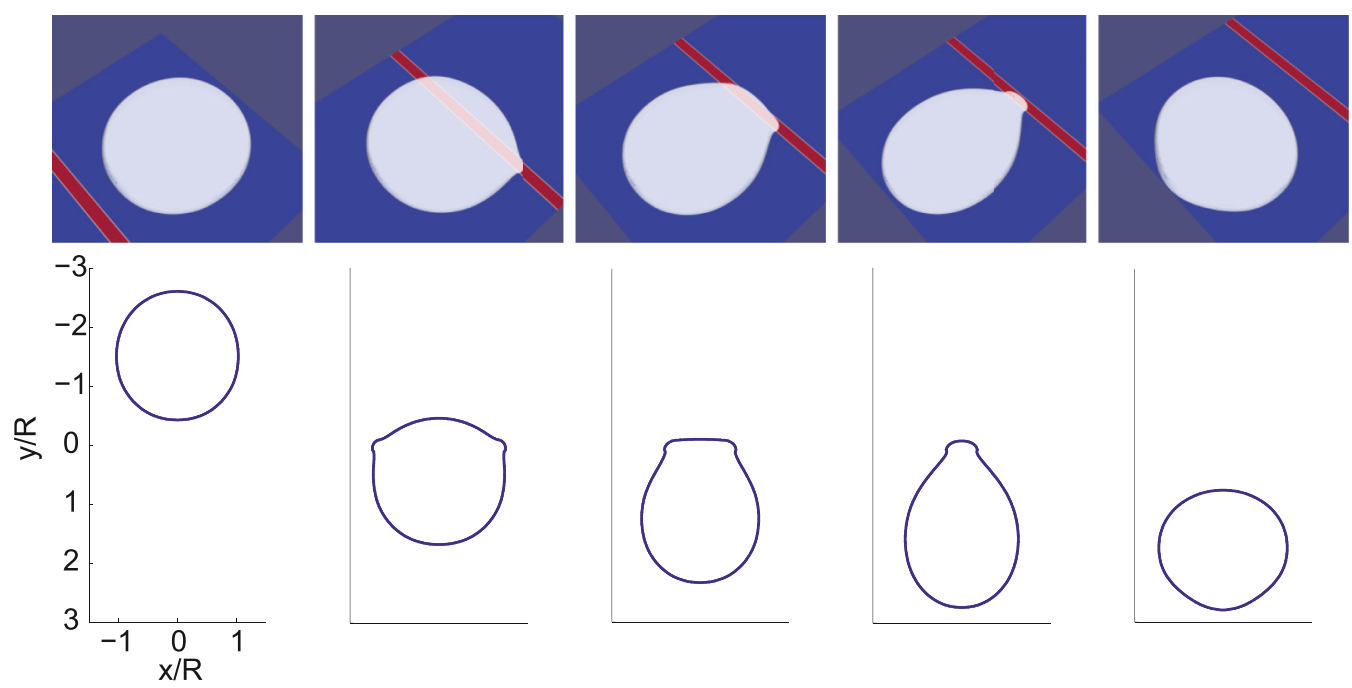

FIG. 4. (Color online) Selected snapshot and corresponding base profile for $\alpha=5^{\circ}, \theta_{\text {trap }}=30^{\circ}$ (top), and $\alpha=7^{\circ}, \theta_{\text {trap }}=45^{\circ}$ (bottom). The wetting defect is represented by the red stripe.

this information for $\theta_{\text {trap }}=45^{\circ}$ and $\alpha=6^{\circ}, 7^{\circ}$ (orange dots, fitted by the red dashed line) to the corresponding energy profile derived from Eq. (11) (blue line). It can be seen that the potential from the numerical simulation is deeper, which is consistent with the drop stretching along the wetting stripe in the 3D model, as observed in Fig. 4. Moreover, the range of the defect in the simulation is larger and skewed towards the escape direction (positive $x$ values). This means that the drop enters the defect approximately as predicted by the analytic model, but on its way out it stretches significantly in the sliding direction, remaining inside the range of the defect for a longer time. Therefore, more kinetic energy will be dissipated while inside the potential well, enhancing the chance of trapping. We can further consider the phase-space plots in Figs. 6(b) and 6(c), which compare the trajectories from the point mass model (blue lines) and the full numerical simulation (red dash-dotted lines) for $\alpha=5^{\circ}$ and $6^{\circ}$. In Fig. 6(b), we observe a substantial agreement: the droplet is captured and reaches approximately the same equilibrium position in both models. We, however, observe more damping in the numerical simulation, as part of the kinetic energy is lost to droplet oscillations. The difference between the two approaches is, however, clear in Fig. 6(c): Under these conditions, according to Fig. 5, the point-mass approach predicts an escape, but the simulation shows trapping. We then see again that the ability of the droplet to stretch extends the effective range of the trap: according to the point-mass model, the center of mass of the droplet exits the defect, but the stretchable droplet is still held back by the trap.

Interestingly, Musterd et al. [6] recently found in a systematic study of the onset of drop sliding on inclined plates that drop deformations can reduce the overall pinning force on surfaces with finite contact angle hysteresis. This reduction was found to be caused by local relaxations of the drop on scales small compared to the drop size. In our present work, the deformation is more global and leads to the opposite effect, i.e., stronger pinning. This suggests the existence of a crossover from reduced pinning to enhanced pinning depending on the 


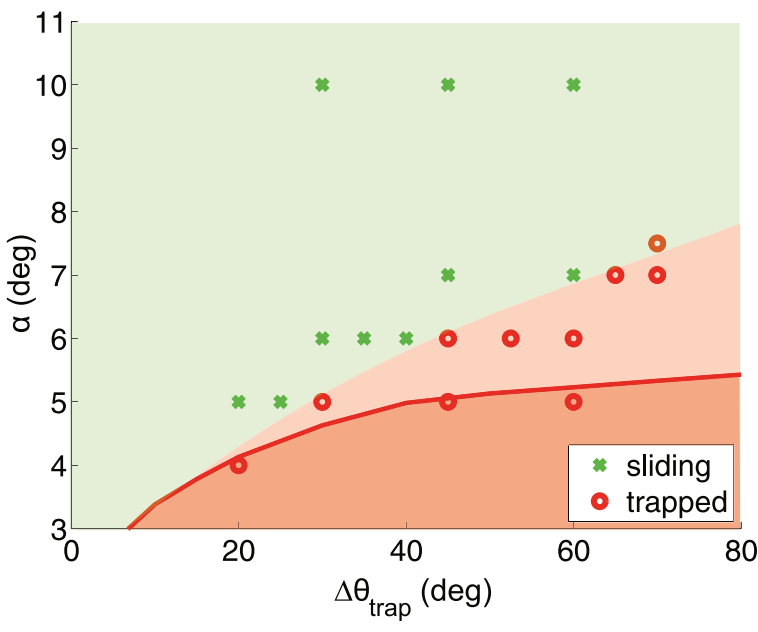

FIG. 5. (Color online) Diagram showing numerical simulations resulting in trapping (red circles) and escaping (green crosses) for a 40- $\mu \mathrm{L}$ water droplet, as a function of the slope $\alpha$ and the strength of the trap, which is proportional to $\Delta \theta_{\text {trap }}=\theta_{0}-\theta_{\text {trap }}$. The darker shade of red represent the trapping region according to the analytic "spherical cap" approximation.

length scale of the heterogeneity as compared to the drop size. It is worth stressing, however, that the stripe defects we consider always fall in the "macroscopic" category. While their width may be small compared to the radius of the drop, they extend across the whole droplet. This results in a coherent deformation of the droplet and a finite energy barrier to the displacement of the drop, as we have seen. Conversely, the relaxation of the droplet in Ref. [6] relies on infinitesimal, quasistatic displacements.

We now discuss how the shape and size of the defect affect the energy landscape experienced by the drop. We start from (a)

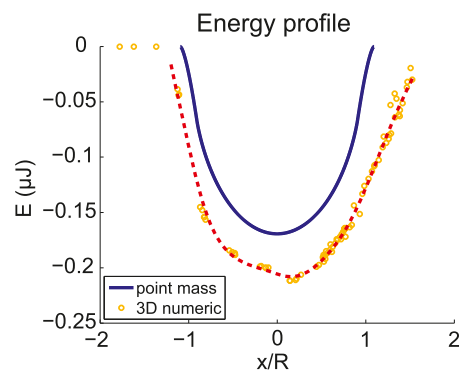

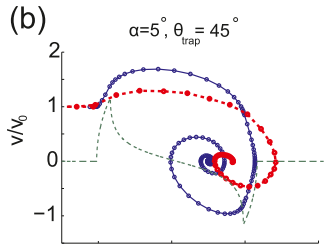

(c)

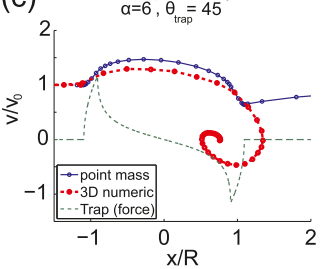

FIG. 6. (Color online) (a) The energy landscape experienced by the sliding drop in the numerical simulation (circles, red fit) is compared to the prediction from the spherical cap approximation (blue solid line). (b) Phase-space plot for $\alpha=5^{\circ}$ and $\theta_{\text {trap }}=45^{\circ}$ : Both the spherical cap model (blue full lines) and the simulation (red thick dashed lines) predict trapping. (c) Phase-space plot for $\alpha=6^{\circ}$ and $\theta_{\text {trap }}=45^{\circ}$ : Thanks to stretching in the sliding direction, the drop is retained in the numerical simulation, while the spherical cap approximation predicts escaping. In (b) and (c), the green dashed line shows the range and magnitude of the force acting on ther drop in the spherical cap approximation (arbitrary units). defects of fixed width $a$ and different contact angle, as sketched in Fig. 7(a). As already reported in Fig. 5, increasing the wettability contrast results in deeper energy wells, while their width is not affected. This is shown in Fig. 7(b), where we plot the energy landscape according to the analytic model. We can alternatively consider traps of fixed wettability and different width $a$ [Figs. 7(c) and 7(d)]. Increasing $a$ results in a larger maximum overlap between the droplet and the stripe. Moreover, the range over which the droplet touches the defect also increases. Therefore, the energy well perceived by the droplet becomes wider and deeper. Another observation is that the assumption of an overall hemispherical shape for the droplets does not hold anymore for wide traps. The drop assumes an overall elongated shape, as can be seen in the insert of Fig. 7(c), and the approximation of Eq. (10) is less accurate. Based on the interfacial area between drop and trap and drop and substrate, we estimate that the energy well in the simulations is approximately $30 \%$ deeper than predicted by the analytic model with hemispherical drops. For each of the trap profiles described, we also performed a phase-space orbits analysis analogous to the one reported in Fig. 6. These results are shown in Fig. 8 and give us a better insight into the dynamics of the interaction.

We clearly notice that the amplitude of the velocity oscillations is larger for deeper energy wells, both if this increase depends on wider traps [Figs. 8(a), 8(c), and 8(e)] or smaller $\theta_{\text {trap }}$ [Figs. 8(b), 8(d), and 8(f)]. Notice also how, in Fig. 8(c), the simulation shows trapping while the analytic model predicts the escape of the droplet. Similarly, in Fig. 8(d), the simulation captures the drop even if its center of mass is outside the nominal width of the trap. These observations are consistent with the discussion of Fig. 6 and constitute further examples of a higher chance of trapping when the internal degrees of freedom of the droplet are taken into account. Another relevant property to consider is the sharpness of the wetting defect. As shown by Joanny and de Gennes [1] this parameter is highly relevant in determining whether a defect pins a contact line or just deforms it. In Fig. 7(e). We then consider a sequence of defects with same surface energy excess but different steepness. According to Eq. (11), we can obtain this by changing the trap width $a$ while keeping $a \cos \theta_{\text {trap }}$ constant. For this class of defects, we observe that large contact angle traps are not as effective in trapping drops, although their action extends over a wider spatial range. This can be explained by looking at Fig. 7(f), where we plot the energy landscape for the droplet in the spherical cap approximation, including the gravity contribution: As the trap gets wider, the energy barrier to the escape of the droplet is progressively smeared out and eventually disappears. It is worth mentioning that the steepness of the defect cannot be increased arbitrarily while retaining the same excess surface energy. This is because the wetting contrast, i.e., the surface energy gain per unit length of the stripe, cannot exceed $\gamma$ (for the system we consider, the maximum wetting contrast is from $90^{\circ}$ to $0^{\circ}$ ). If the maximum wetting contrast is reached, further reducing the stripe width results in a reduced trapping potential.

Finally, we would like to assess whether the nature of the defect (chemical, electrical) affects the trapping. To do so, we compare our simulations to previously reported trapping experiments on electrowetting defects [16]. We use 


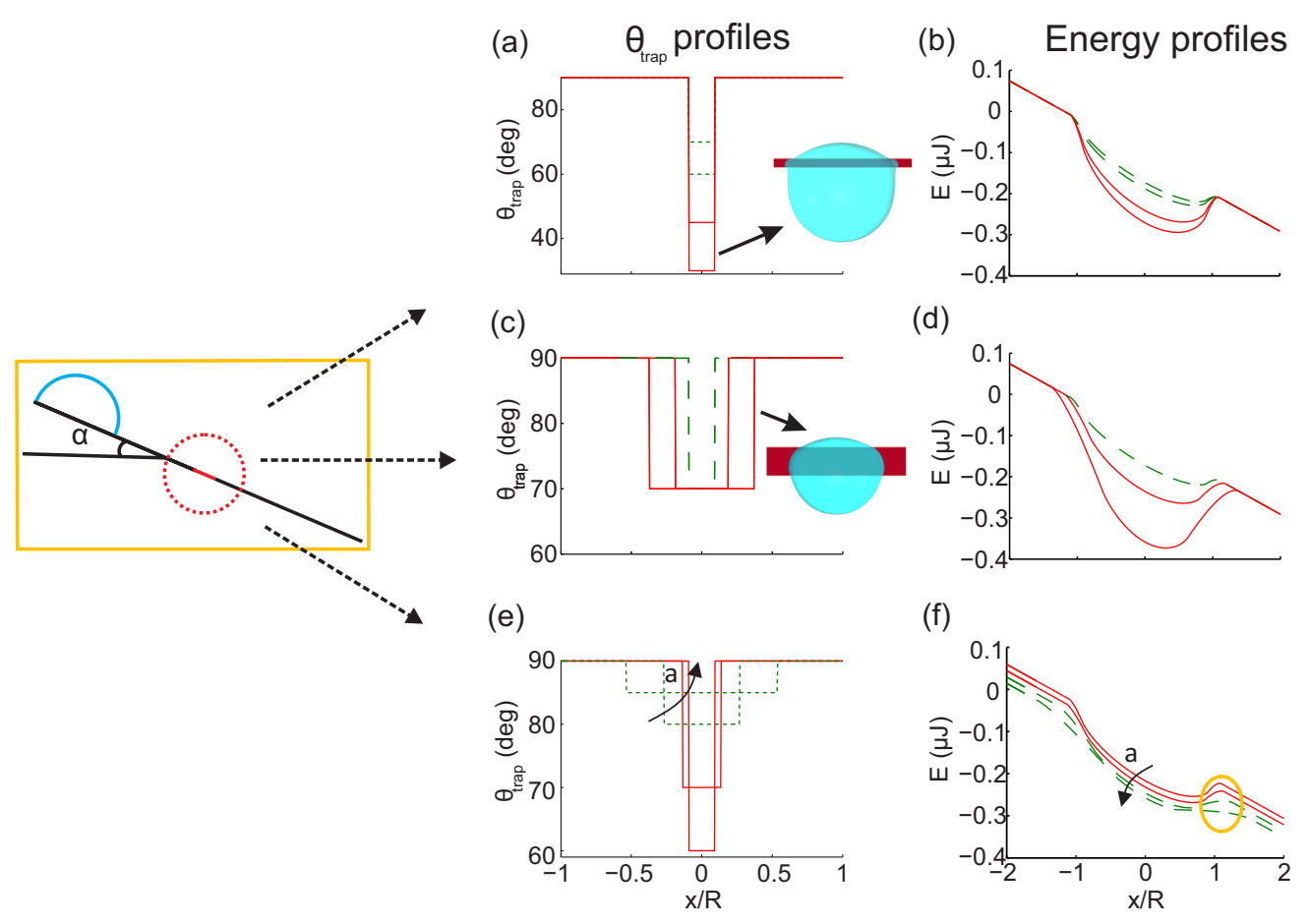

FIG. 7. (Color online) A comparison of defects of different size and shapes. Contact angle profiles for wetting defects of (a) fixed width $a$ and different contact angle $\theta_{\text {trap }}$. (c) fixed contact angle $\theta_{\text {trap }}$ and variable trap width $a$. (e) fixed excess energy, $a \cos \theta_{\text {trap }}=$ const. The shape of the trapped droplet for a wide and thin trap are also shown as an insets. In all pictures, red solid lines represent simulations in which the droplet is captured, while for green dashed lines the droplet is only slowed down. [(b) and (d)-(f)] Corresponding energy landscapes in the spherical cap approximation, including the gravity contribution for $\alpha=5^{\circ}$. The profiles in (f) are shifted to ease the visualization.

the characteristic scales from the spherical cap approximation to normalize our numerical results [Fig. 9(b)] and compare them to experimental data [Fig. 9(a)] from Ref. [16].

On the $y$ axis we plot the normalized driving force $\tilde{F}_{g}=\frac{F_{g}}{F_{\text {trap }}}$, where $F_{\text {trap }}$ is the maximum trapping force acting on the drop according to Eq. (12). If $\tilde{F}_{g}>1$ (above the gray line in Fig. 9), gravity is able to pull the drop out of the potential minimum regardless of its kinetic energy. For $\tilde{F}_{g} \leqslant 1$, inertia plays a relevant role: To analyze it, on the $x$ axis we plot the product $Q=\frac{1}{2} \omega \tau_{\text {visc }}$ of the characteristic viscous time $\tau_{\text {visc }}=\frac{2 m}{\lambda}$ and the "trap frequency" $\omega=\sqrt{\frac{F_{\text {trap }}}{m R}}$. If $Q \ll 1$, energy dissipation happens on a much smaller time scale than the drop-trap interaction. Kinetic energy is then lost while the drop crosses the trap, and we observe trapping. If, instead, $Q \gg 1$, the dissipation is slow on the time scale that the drop spends inside the potential well. The drop can then escape due to its stored kinetic energy. The black line in the plot shows the boundary between the two regimes in the spherical cap approximation. Once the numerical results are normalized according to these units, we observe a good quantitative agreement to the experimental results described by 't Mannetje [16]. This result is rather interesting, as it corroborates the findings of 't Mannetje and colleagues that the trapping of droplets on diverse obstacles (chemical, electric, or topographical) can be described by a unified approach. Concerning the scope of this paper, we stress that both the numerical and experimental data show an excess trapping at large $Q$, if compared to the spherical cap approximation. This is consistent with the deformation of the droplet enhancing the chance of trapping, as we discussed.

\section{CONCLUSIONS AND OUTLOOK}

In this paper, we introduce a three-dimensional numerical model to analyze the dynamic interaction of sessile drops with tunable wetting defects. By comparison with a simplified analytic model, we conclude that droplet deformations enhance the effective strength and range of the trap. We also analyze the effect of the strength, shape, and steepness of the defect and observe that shallow traps are less effective at retaining drops in comparison with localized defect of the same excess surface energy. Upon normalization, our data show the same scaling of previously reported electrowetting experiments: On one hand, this validates our model, which can be employed to design and test steering and trapping devices [15] based on electrowetting or chemical patterning. On the other hand, our analysis supports the idea, described in Ref. [16], of an universal drop-trap interaction dynamics, independent on the details of the defect.

\section{ACKNOWLEDGMENTS}

The authors thank Ivo Roghair for useful discussions on the implementation of the OPENFOAM model and Dirk van den Ende for valuable suggestions concerning the analytic description of the system. This research is supported by the Dutch Technology Foundation STW, which is part of the Netherlands Organisation for Scientific Research (NWO), and which is partly funded by the Ministry of Economic Affairs. 

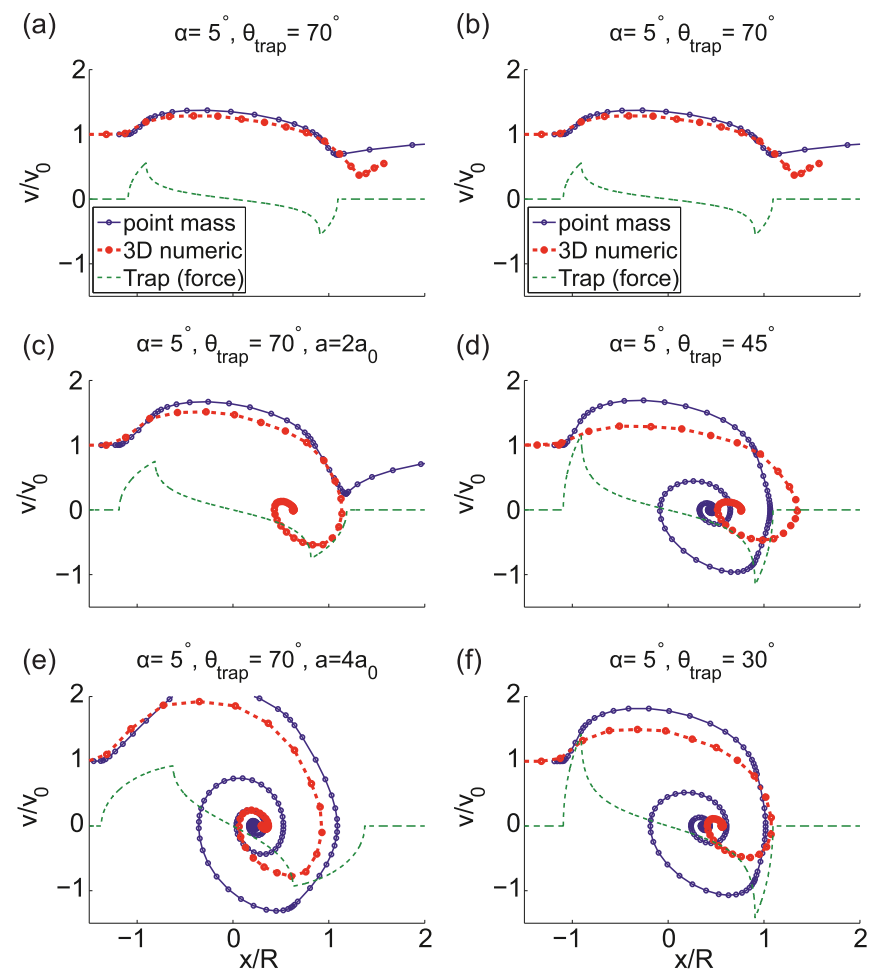

FIG. 8. (Color online) Phase-space plots for traps of increasing width $a$ and fixed $\theta_{\text {trap }}[(\mathrm{a}),(\mathrm{c})$, and (e)] and traps of fixed width $a$ and different $\theta_{\text {trap }}[(\mathrm{b}),(\mathrm{d})$, and (f)]. The colors are the same as in Figs. 6(b)-6(c). As discussed in the main text, deeper energy wells (see Fig. 7) result in wider oscillations of the droplet velocity. Notice how in (c), once again, the numerical simulation shows trapping, while the analytic model predicts escaping.

\section{APPENDIX A: MESH DESIGN AND CONVERGENCE CHECK}

Even exploiting the symmetry of the system with respect to the midplane of the drop, the three-dimensional, timedependent simulations we carry out are quite expensive in (a)

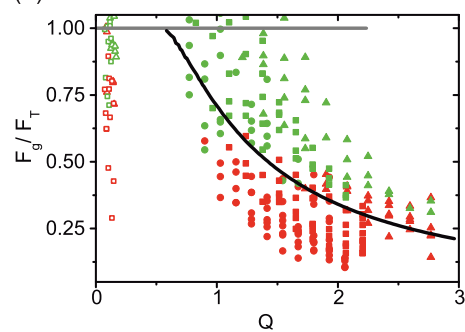

(b)

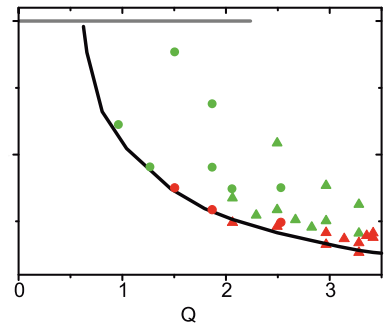

FIG. 9. (Color online) Normalized trapping and escaping landscape for (a) the experiments in Ref. [16] and (b) the numerical simulations in this work. As usual, (light) green represents sliding droplets and (dark) red trapped ones. In (b), full dots represent simulations with linearly varying contact angle, as in Fig. 11, and triangles are traps with a constant static contact angle $\theta_{\text {trap. }}$ In both cases, the black line represents the transition between trapping and escaping in the spherical cap approximation, which underestimates the trapping at large $Q$.

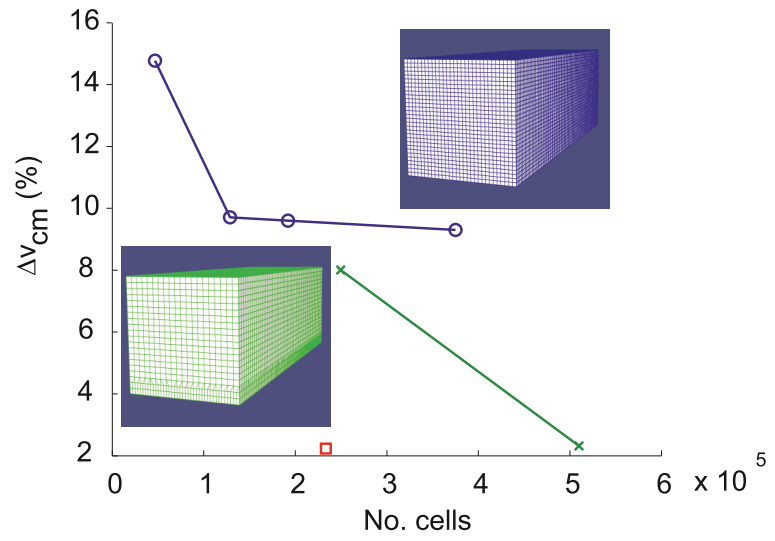

FIG. 10. (Color online) Convergence plot for freely sliding drops. The blue circles are obtained for homogeneous resolution meshes (as in the sketch in the top right corner). The green crosses are obtained for locally refined meshes (sketched in the lower left corner). The red square represents a reduced domain size, still showing good agreement with the high-resolution simulation.

terms of computation time. We therefore took some measures to optimize the execution, while trying to minimize the size of the computational mesh. First, we obtain a steadystate sliding drop profile only once for each slope $\alpha$ and subsequently restart the simulation from the steady-state sliding configuration, adding each time a different trap on the path of the drop. We also employ orthogonal meshes which are easily parallelized. We validated our mesh choice by comparing the velocity of a $40-\mu \mathrm{L}$ droplet for increasingly high mesh resolution, while it slides down a slope of $\alpha=10^{\circ}$. In Fig. 10, we plot the cumulative deviation in the drop velocity over time steps $t_{i} \Delta v_{\text {c.m. }}=\sqrt{\sum_{i=0}^{i=i_{\max }} \frac{\left[v\left(t_{i}\right)-v_{\text {ref }}\left(t_{i}\right)\right]^{2}}{v_{\text {ref }}^{2}\left(t_{i}\right)}}$ with respect to $v_{\text {ref }}$, the velocity obtained at the highest $(x, y, z)$ resolution considered, $(300 \times 100 \times 100)$ cells. If the mesh resolution is homogeneously increased (blue circles), we observe a poor convergence to the high-resolution result. If we instead locally refine the mesh in proximity of the substrate (green crosses), we observe a much faster convergence as a function of the number of cells employed. This is not surprising, as we expect the dynamic of the system to be significantly affected by the contact line motion, i.e., the substrate-drop interaction. We therefore selected the best locally refined mesh in Fig. $10\left(\simeq 5 \times 10^{5}\right.$ cells for a $(1.5 \mathrm{~cm} \times 0.5 \mathrm{~cm} \times 0.5 \mathrm{~cm})$ domain, $2 \%$ deviation from the high-resolution result) as our discretization scheme. The length of the domain is chosen to avoid self-interference of the drop with itself through the periodic boundary. We also tested a smaller domain with the same resolution as the optimal mesh $(1 \mathrm{~cm} \times 0.4 \mathrm{~cm} \times 0.5 \mathrm{~cm}$, red square in Fig. 10), observing no significant discrepancy. The selected mesh is a compromise between execution time and precision: The three-dimensional nature of the system makes it computationally expensive to achieve the typical resolution of a two-dimensional or axisymmetric simulation. However, the consistent agreement of our simulations with experiments for freely sliding drops and drop-trap interaction supports our discretization choices. 

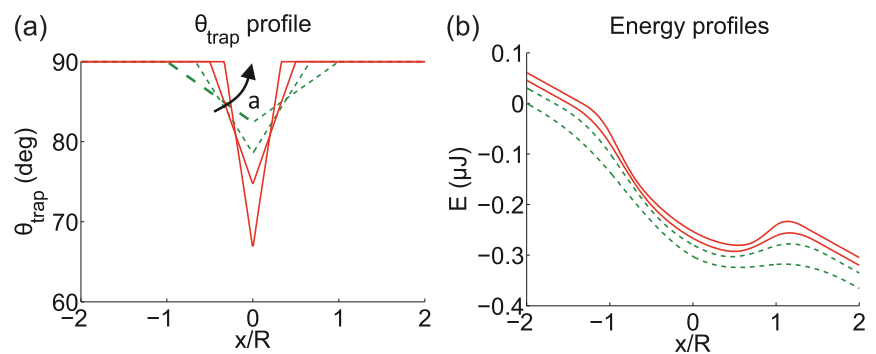

FIG. 11. (Color online) A comparison of defects of different size and shapes for $a \cos \theta_{\text {trap }}=$ const (see also Fig. 7). In both pictures, red solid lines represents simulations in which the droplet is captured, while for green dashed lines the droplet is only slowed down. (a) Contact angle profiles. (b) Corresponding energy landscapes in the spherical cap approximation for $\alpha=5^{\circ}$.

\section{APPENDIX B: LINEARLY VARYING CONTACT ANGLE TRAPS}

Our choice of a constant contact angle inside the trap allowed us to simplify the energy calculations in Eqs. (11) and (12). However, our numerical model and analytic considerations hold quite generally. In this appendix we briefly discuss a different wettability profile, shown in Fig. 11(a), which is obtained by choosing the following parametrization for the Young contact angle:

$$
\cos \theta(x)=\left\{\begin{array}{ll}
\left(1+\frac{2 x}{a}\right) \cos \theta_{\text {trap }} & -\frac{a}{2}<x<0 \\
\left(1-\frac{2 x}{a}\right) \cos \theta_{\text {trap }} & 0<x<\frac{a}{2} \\
0 & \text { otherwise }
\end{array} .\right.
$$

The equivalent of Eq. (10) for such traps reads:

$$
\begin{aligned}
\Delta U(x)= & U_{0}-2 \gamma \cos \theta_{\text {trap }}\left[\int_{0}^{\frac{a}{2}} \sqrt{R^{2}-(x+u)^{2}}\left(1-\frac{2 u}{a}\right)\right. \\
& \left.\times d u+\int_{-\frac{a}{2}}^{0} \sqrt{R^{2}-(x+u)^{2}}\left(1+\frac{2 u}{a}\right) d u\right] .
\end{aligned}
$$

The integration over the width of the stripe is due to the fact that the contact angle is not constant: therefore, each infinitesimal stripe of width $d u$ and length $\sqrt{R^{2}-(x+u)^{2}}$ contributes to $\Delta U(x)$ for $\gamma \cos \theta_{\text {trap }}\left(1 \pm \frac{2 u}{a}\right)$. The results we observe are consistent with the traps discussed in the main text. In Fig. 11, for example, we consider traps with $a \cos \theta_{\text {trap }}=$ const: The corresponding energy profiles in the spherical cap approximation are shown in Fig. 11(b). We once again observe the smearing out of the energy well as the width of the trap is increased and the wettability reduced, as discussed in the main text and Fig. 7.
[1] J. Joanny and P.-G. De Gennes, A model for contact angle hysteresis, J. Chem. Phys. 81, 552 (1984).

[2] P.-G. De Gennes, F. Brochard-Wyart, and D. Quéré, Capillarity and Wetting Phenomena: Drops, Bubbles, Pearls, Waves (Springer, Berlin, 2004).

[3] V. E. Dussan and R. T.-P. Chow, On the ability of drops or bubbles to stick to non-horizontal surfaces of solids, J. Fluid Mech. 137, 1 (1983).

[4] M. Santos, S. Velasco, and J. White, Simulation analysis of contact angles and retention forces of liquid drops on inclined surfaces, Langmuir 28, 11819 (2012).

[5] C. Semprebon and M. Brinkmann, On the onset of motion of sliding drops, Soft Matter 10, 3325 (2014).

[6] M. Musterd, V. van Steijn, C. R. Kleijn, and M. T. Kreutzer, Droplets on inclined plates: Local and global hysteresis of pinned capillary surfaces, Phys. Rev. Lett. 113, 066104 (2014).

[7] U. Thiele and E. Knobloch, Driven drops on heterogeneous substrates: Onset of sliding motion, Phys. Rev. Lett. 97, 204501 (2006).

[8] H. Kusumaatmaja, J. Leopoldes, A. Dupuis, and J. Yeomans, Drop dynamics on chemically patterned surfaces, Europhys. Lett. 73, 740 (2006).

[9] R. Ledesma-Aguilar, A. Hernandez-Machado, and I. Pagonabarraga, Dynamics of gravity driven three-dimensional thin films on hydrophilic- hydrophobic patterned substrates, Langmuir 26, 3292 (2010).

[10] D. Herde, U. Thiele, S. Herminghaus, and M. Brinkmann, Driven large contact angle droplets on chemically heterogeneous substrates, Europhys. Lett. 100, 16002 (2012).
[11] M. Sbragaglia, L. Biferale, G. Amati, S. Varagnolo, D. Ferraro, G. Mistura, and M. Pierno, Sliding drops across alternating hydrophobic and hydrophilic stripes, Phys. Rev. E 89, 012406 (2014).

[12] M. E. Kavousanakis, C. E. Colosqui, I. G. Kevrekidis, and A. G. Papathanasiou, Mechanisms of wetting transitions on patterned surfaces: Continuum and mesoscopic analysis, Soft Matter 8, 7928 (2012).

[13] A. Lafuma and D. Quéré, Superhydrophobic states, Nat. Mater. 2, 457 (2003).

[14] R. Dangla, S. Lee, and C. N. Baroud, Trapping microfluidic drops in wells of surface energy, Phys. Rev. Lett. 107, 124501 (2011)

[15] R. de Ruiter, A. M. Pit, V. M. de Oliveira, M. H. G. Duits, and D. van den Ende, and F. Mugele, Electrostatic potential wells for on-demand drop manipulation in microchannels, Lab Chip 14, 883 (2014).

[16] S. G. Dieter't Mannetje, R. Lagraauw, S. Otten, A. Pit, C. Berendsen, J. Zeegers, D. van den Ende, and F. Mugele, Trapping of drops by wetting defects, Nat. Commun. 5, 3559 (2014).

[17] C. Priest, R. Sedev, and J. Ralston, Asymmetric wetting hysteresis on chemical defects, Phys. Rev. Lett. 99, 026103 (2007).

[18] D. t Mannetje, A. Banpurkar, H. Koppelman, M. H. Duits, D. van den Ende, and F. Mugele, Electrically tunable wetting defects characterized by a simple capillary force sensor, Langmuir 29, 9944 (2013).

[19] S. S. Deshpande, L. Anumolu, and M. F. Trujillo, Evaluating the performance of the two-phase flow solver interFoam, Comput. Sci. Discov. 5, 014016 (2012). 
[20] M. Sussman, P. Smereka, and S. Osher, A level set approach for computing solutions to incompressible two-phase flow, J. Comput. Phys. 114, 146 (1994).

[21] R. Cox, The dynamics of the spreading of liquids on a solid surface. Part 1. Viscous flow, J. Fluid Mech. 168, 169 (1986).
[22] P. Petrov and I. Petrov, A combined molecular-hydrodynamic approach to wetting kinetics, Langmuir 8, 1762 (1992).

[23] O. Ubbink, Numerical prediction of two fluid systems with sharp interfaces, Ph.D. thesis, University of London, 1997. 